\title{
Quality study of hand made brick-DK using neutron radiography technique
}

\author{
M. K. Alam ${ }^{1 *}$, M. R. Islam ${ }^{2}$, S. Saha ${ }^{1}$, M.N. Islam ${ }^{1}$ and S. M. Azaharul Islam² \\ ${ }^{1}$ Institute of Nuclear Science and Technology, AERE, Savar, Dhaka \\ ${ }^{2}$ Department of physics, Jahangirnagar University, Savar, Dhaka
}

\begin{abstract}
Neutron radiography is a powerful non-destructive testing (NDT) technique for internal evaluation of materials, such as voids/cavity, cracks, homogeneity, water absorption behavior, etc. It involves attenuation of a neutron beam by an object to be radiographed and thus to make the registration of the attenuation process (as an image) on a film or video. In the present investigation neutron radiography (NR) imaging technique has been adopted to study the quality like homogeneity, porosity, water penetrating height, behavior of incremental intrusion area, initial rapid absorption (IRA) of the brick-DK sample. Thermal neutron radiography facility installed at the tangential beam port of $3 \mathrm{MW}$ TRIGA MARK-II reactor is used in this study. In this cases optical density or gray values of the neutron radiographic images of the sample is measured. From this measurement it is found that the contents of the sample is not uniformly distributed all over the sample which indicates the presence of large number of internal porosity, at the two edges water uptake is slightly poorer than the middle part and initial rapid absorption is very high. Water penetrating inside the sample is faster. The results obtained and conclusion made in this study can only be compared to the properties of bricks produced under similar conditions with similar raw materials.
\end{abstract}

Keywords: Neutron radiography; IRA; Water penetration; Homogeneity

\section{Introduction}

Neutron Radiography (NR) is a technique of making a picture of the internal details of an object by the selective absorption of a neutron beam by the object. NR uses the basic principles of radiography whereby a beam of radiation is modified by an object in its path and the emergent beam is recorded on a photo film (detector). In general, the Radiography technique is nothing but it is a simple process of exposing some objects to an X-ray, gamma-ray, neutron beam and some other types of radiation and then attenuated outgoing beam from the object is passing through a special type of photographic film to form images of the objects on the radiographic film or detector. Also it is called a nondestructive testing (NDT) (Berger, 1964) and evaluation technique of testing non-nuclear and nuclear materials and industrial products. There are large numbers of brick kiln present in Bangladesh. These are 150 years old conventional drum and fixed chimney kiln brick field. Bangladesh produced large amount of clay fired bricks, is estimated to have more than 100,000 brick kilns, producing about 150-200 billion bricks annually (Janakantha, 2012), employing about 10 million workers and consuming about 25 million tons of coal annually.
It is also a powerful non-destructive testing technique for internal evaluation of materials, such as voids/ cavity, cracks, homogeneity (Alam and Khan, 2006), porosity etc. This method is also used for characterizing the internal structure, internal defects in materials like ceramics (Alam, 2005), tiles (Alam et al., 2007) and different building industries (Islam et al., 2000) etc. The technique is also adopted for the study of water absorption behavior in biopol composite, jute-reinforced-biopol composite (Alam et al., 2006) and wood plastic composites (Alam et al., 2003) etc. Clay is a widely available raw material that survives very well in its fired form. Clay bricks have been found in the ruins of ancient civilizations (Maithel et al., 2012). Bhatnagar et al. (2002) saw that properties of these bricks are affected as a result of physical, chemical and mineralogical changes (Bhatnagar and Goel, 2002; Cultrone et al., 2004). Mbumbia et al. (2000) investigated that compressive strength and water absorption are two major physical properties of brick that are good predictors of bricks ability to resist cracking of face.

Water absorption is a measure of available pore space and is expressed as a percentage of the dry brick weight. It is affected by properties of clay, method of manufacturing and

\footnotetext{
*Corresponding author. e-mail: alam1964@yahoo.co.in
} 
degree of firing. Water absorption capacity of the brick affects the surface finishing of the brick-laid wall (Presertsan and Theppaya, 1995; Part-1, 1986; Marrusin, 1985).

Neutron radiograph gives the information of the internal structure of an object; can detect light elements, which have large neutron absorption cross-sections like hydrogen and boron; completely complementary to other NDT techniques, like X-ray or gamma ray radiography. The atoms of the object material scattered or absorbed the radiation and so the beam reaching the detector shows an intensity/gray value pattern representative of the internal structure of the object (Hardt and Rotterger, 1981). The beam, which remains after passing through, enters a detector that registers the fraction of the initial radiation intensity that has been transmitted through each point of the object. Any in-homogeneity in the object on an internal defect (such as voids, cracks, porosity, inclusion, corrosion etc.) will show up as change in gray value/radiation intensity reaching the detector. Aim of the present work is as follows:

(i) Optimization of irradiation/exposure time.

(ii) Determination of elemental distribution/homogeneity in the sample.

(iii) Determination of incremental intrusion area of water/water penetrating height and penetrating shape/behavior.

\section{Materials and methods}

\section{(a) Sample collection and preparation}

The sample (brick-DK) is collected from Lucky (Home) bricks factory located at Dautia, Kalampur, Dhamrai, Dhaka. Then the sample is polished manually by using sand paper, cement block and diamond cutter to make it as a test object. After that the sample was dried at day light/dryer machine at $65^{\circ} \mathrm{C}$ until its constant weight is attained. The actual size, shape and weight of this sample is $24.300 \times 11.535 \times 6.460$ $\mathrm{cm}^{3}$, rectangular and $3120 \mathrm{gm}$, respectively.

\section{(b) Loading converter foil and film in the NR-cassette}

A thin converter (gadolinium metal foil of $25 \mu \mathrm{m}$ thickness) was placed at the back of the X-ray industrial film. The loading of the X-ray industrial film (Agfa structurix D4DW) into the NR cassette $(18 \mathrm{~cm} \times 24 \mathrm{~cm})$ is a simple procedure (Bouma, 1969) which requires only a little practice to be carried out easily in the darkroom. Loading should be done near the cupboard where the film is stored. The "dry" table should only be used for this work to prevent any contact of developing liquids with the unexposed loading film. Few steps are being followed to place the industrial X-ray film into the NR cassette to protect the film against day light and lamplight. The best way to load the cassette is to open the NR cassette

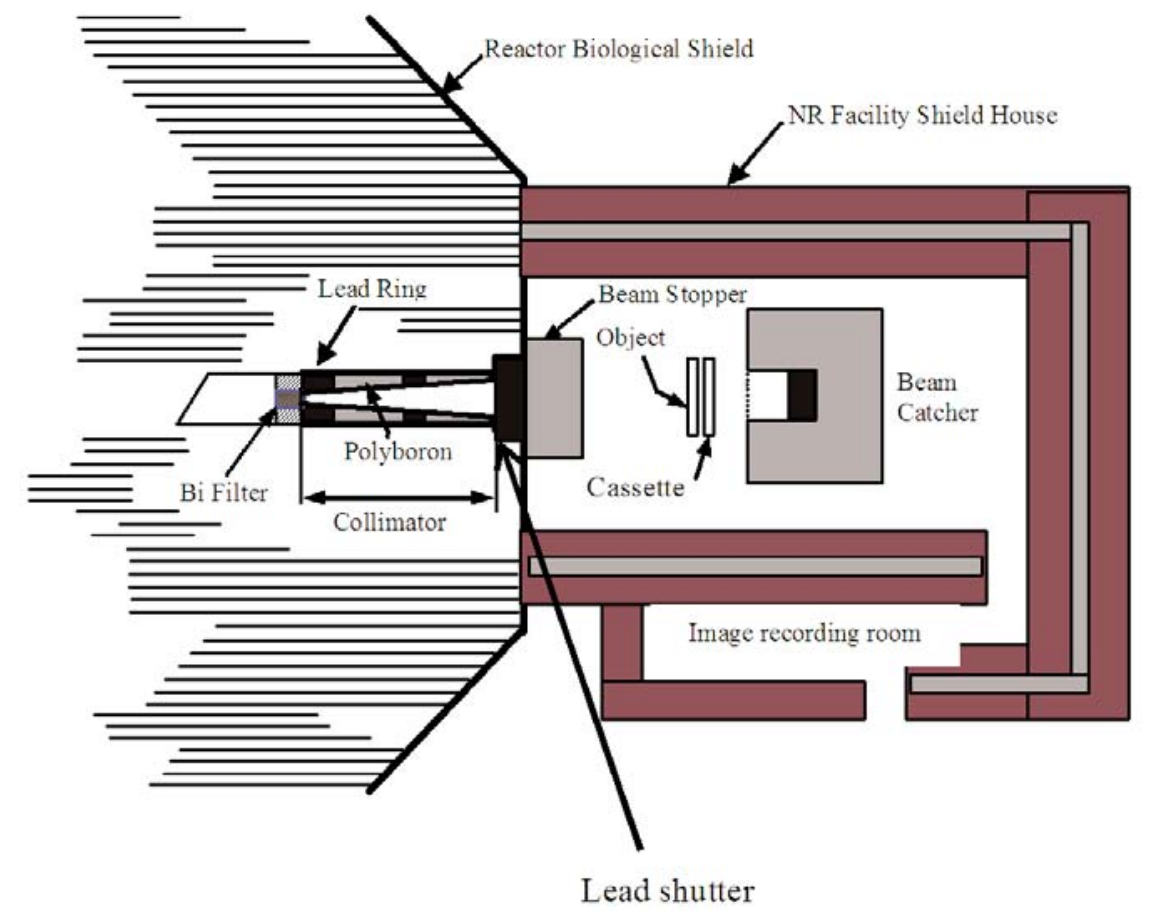

Fig. 1. Schematic diagram of the neutron radiography facility 
when it lies on the "dry" table. While using proper illumination only, a film still in the interleaving paper is carefully drawn out from the box holding the film between thumb and finger and putting the film in the cassette. It is very convenient and easy to load in the dark room.

\section{(c) Placing of sample and the NR-cassette}

The sample is placed in close contact with the NR cassette and directly on the sample holder table. The NR cassette is placed on the cassette holder table. Both of NR cassette and sample are placed in front of the neutron beam (diameter $30 \mathrm{~cm})$.

\section{(d) Determination of neutron beam exposure time}

Exposure means passing of neutron beam through a sample and holding it onto a special film (X-ray industrial film) in order to create a latent image of an object in the emulsion layers of that film. Of course, this discussion is confined only to direct contact radiographic film (film in close contact with the Gd converter foil). Exposure time differs for different samples, depending on the intensity of the neutron beam, density and thickness of the sample and neutron cross-section. The optimum exposure time of the sample was determined by taking a series of experiments/radiographs with different exposure time, while the reactor was operated at $250 \mathrm{~kW}$. For the present experiment it was found that the optimum exposure time is 60 minutes. The sample was then irradiated for that optimum period to obtain good neutron radiographs (Fig. 2).

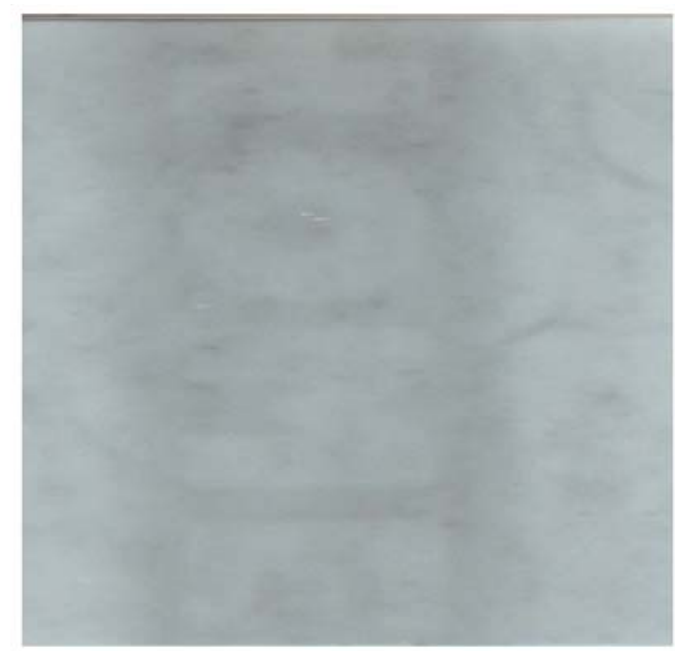

Fig. 2. Radiograhic image of the dry sample (partial)

\section{(e) Immersion procedure of the brick sample}

The sample is placed in a plastic pan and a constant height of $2.0 \mathrm{~cm}$ water level is maintained. The water level is observed very carefully and extra water was added to maintain water level during the immersion time. After time of interest (TOI) such as 5, 10, 15 and 20 minutes brick sample was taken out from the pan and water from the surface of the sample was removed by tissue paper.

(f) Obtaining radiographic images of the sample

\section{i) Irradiation:}

After completion of the required steps (a-e) the neutron beam was exposed by removing the wooden plug, lead plug and beam stopper from the front side of the collimator. After each immersion time sample was then irradiated for the optimum time (60 $\mathrm{min})$ one by one.

\section{ii) Developing}

Development is an image processing technique by which the latent image recorded during the exposure of the material is converted into a silver image (Bjelkhagen, 1993). Developing process is completed at $20^{\circ} \mathrm{C}$ for 05 minutes.

iii) Fixing

When the development is completed, this photographic film must be rinsed/washed in water, after which it is treated in a fixation bath. The fixation solution will dissolve the unexposed silver-halide crystals leaving only the silver grains in the gelatin. The fixing is completed within 05 minutes and maintaining the fixture temperature at $20^{\circ} \mathrm{C}$.

\section{iv) Washing}

In between developing and fixing the radiographic film, it is necessary to wash for 1 minute at flowing tap water.

\section{v) Final washing}

The silver compound which was formed during the fixing stage must be removed, since they can affect the silver image at the latter stage. For this reason the film must be washed thoroughly in flowing tap water for 15 (appx.) minutes after completion of developing and fixing process.

\section{vi) Drying}

After the final washing, the films were dried by clipping in a hanger at fresh air/ or in a drying cabinet. 


\section{Mathematical formulation}

Any in homogeneity in the object or internal defects e.g. voids, cracks, porosity/homogeneity or inclusion will show up as a change in gray value/radiation intensity reaching the detector. Irradiation intensity varies after passing through an object under examination. The intensity/gray value variation obeys the general attenuation law (Norris et al., 1996) applicable for X-rays, gamma rays or neutrons:

$$
\mathrm{I}=\mathrm{I}_{0} \mathrm{e}^{-\mu \mathrm{x}}
$$

Where,

$$
\begin{aligned}
& I_{0}=\text { intensity of the incident neutron beam, } \\
& I=\text { intensity of the emergent beam from the object, } \\
& \mu=\text { neutron attenuation coefficient, } \\
& x=\text { thickness of the test object. }
\end{aligned}
$$

The attenuated neutrons beam enters the detector that resists the fraction of initial radiation intensity that has been transmitted by each point in the object and is then recorded in the radiographic film i.e. image detector.

\section{i) Optical density measurement}

The neutron intensity before reaching the brick sample (object) is different from the intensity of the neutron after passing through it. The relationship between these two intensities is expressed through the equation (Norris et al., 1996)

$$
\mathrm{I}=\mathrm{I}_{0} \mathrm{e}^{-\mu \mathrm{x}}
$$

Where, $\mathrm{e}=$ base of natural logarithms, $\mathrm{x}=$ thickness of the object, $\mu=$ linear neutron attenuation coefficient, $\mathrm{I}$ and $\mathrm{I}_{0}$ are the neutron intensity after passing through the object and the neutron intensity incident on the object, respectively.

The mathematical expression for the optical density (Harms and Wyman, 1986) at a point of the film/NR image, D is given by:

$\mathrm{D}=\ln \left(\mathrm{A}_{0} / \mathrm{A}\right)$

Here, $\mathrm{A}_{0}=$ response of densitometer without the sample image and $\mathrm{A}=$ response of densitometer with the sample image.

The density of film is measured with an optical densitometer (Model 07-424, S-23285, Victoreen Inc. USA) (Islam et al., 2000). A small beam of light from the light source passes through the film area which is measured by densitometer. On the other side of the film, a light sensor (photocell) converts the penetrated light into an electrical signal. A special circuit performs a logarithmic conversion on the signal and displays the results in density units.

The primary use of densitometers in a clinical facility is to monitor the performance of film processors. Actually, optical density is the darkness, or opaqueness, of a transparency film and is produced by film exposure and chemical processing. An image contains areas with different densities that are viewed as various shades of gray.

\section{ii) Gray value}

The visual appearance of an image is generally characterized by two properties such as brightness and contrast. Brightness refers to the overall intensity level and is therefore influenced by the individual gray-level (intensity) values of all the pixels within an image. Since a bright image (or sub image) has more pixel gray-level values closer to the higher end of the intensity scale, it is likely to have a higher average intensity value. Contrast in an image is indicated by the ability of the observer to distinguish separate neighboring parts within an image. This ability to see small details around an individual pixel and larger variations within a neighborhood is provided by the spatial intensity variations of adjacent pixels, between two neighboring sub images, or within the entire image. Thus, an image may be bright (due to, for example, overexposure or too much illumination) with poor contrast if the individual target objects in the image have optical characteristics similar to the background. At the other end of the scale, a dark image may have high contrast if the background is significantly different from the individual objects within the image, or if separate areas within the image have very different reflectance properties. For a captured image with maximum and minimum gray-level values gmax and gmin, and using the sinusoidal image intensity shown in Fig. 3. Image contrast modulation and mean brightness are given by the following equation:

\section{Contrast modulation $=\left[\mathrm{g}_{\max }-\mathrm{g}_{\min }\right] /\left[\mathrm{g}_{\max }+\mathrm{g}_{\min }\right]$}

Although the intensity distribution within any real-life image is unlikely to be purely sinusoidal, these definitions provide a basis for comparison. For example, an image that contains pixels with brightness values spread over the entire intensity scale is likely to have better contrast than the image with pixel gray-level values located within a narrow range. The relationship between the intensity spread at the pixel level 


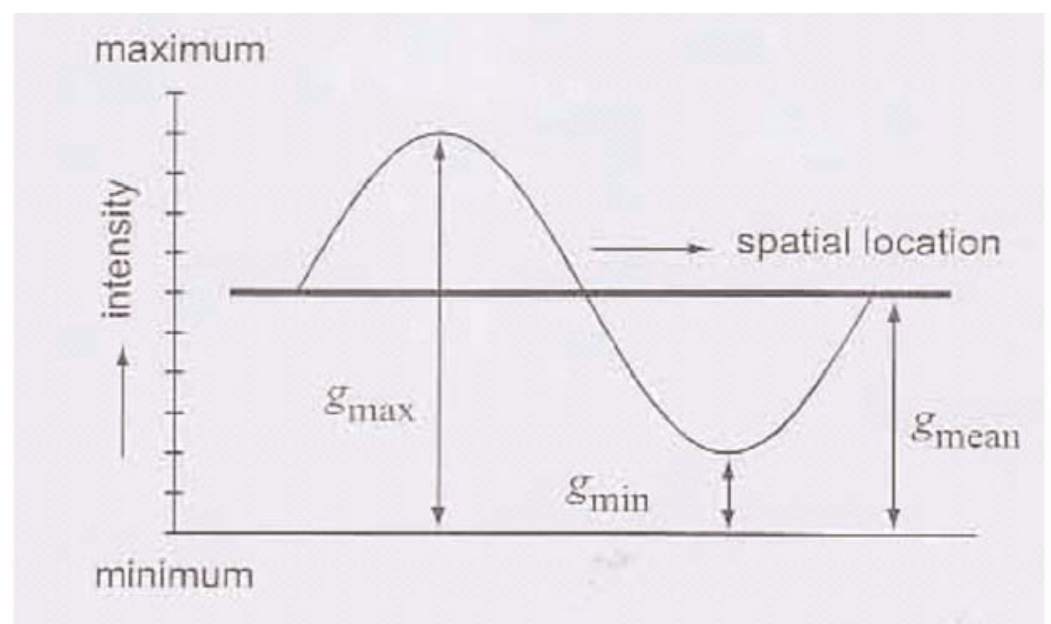

Fig. 3. Conceptual sinusoidal intensity line profile along the horizontal axis
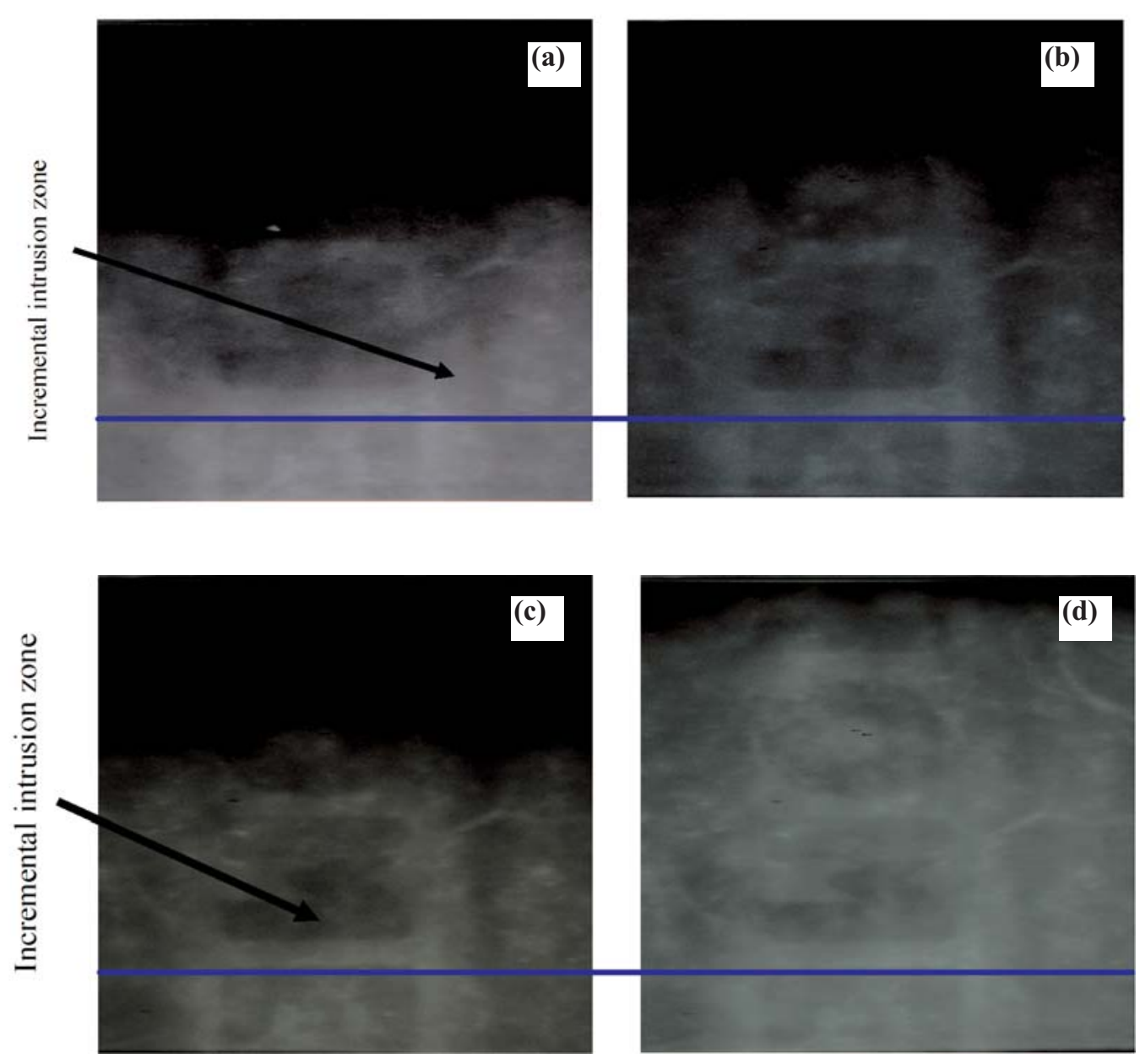

Fig. 4. Radiographic image of the brick-DK sample at different immersion time for (a) 5 minutes (b) 10 minutes (c) 15 minutes and (d) 20 minutes with indicating the water incremental intrusion zone. 
and the overall appearance of an image provides the basis for image enhancement by gray-level transformation. The terms gray value and intensity are used synonymously to describe pixel brightness.

\section{Results and discussion}

Actually, the specific relationship between the shades of gray or density and exposure depends on the characteristics of the film emulsion and the processing conditions. The quality of a brick samples depends on the proper distribution of the contents, porosity, hardiness, water penetration/ absorption behavior etc. in the sample. Fig. 5 shows that gray value/intensity varies with the pixel distance. The gray value/intensity is different at different points. The porosity, elemental distribution of the samples depends on the gray value/intensity and has been studied by measuring the variation of gray value/intensity from the neutron radiographic images of the sample. Variation of gray values of the radiographic images of the samples indicates that the constituent components of the samples are not uniformly distributed and having internal porosity.

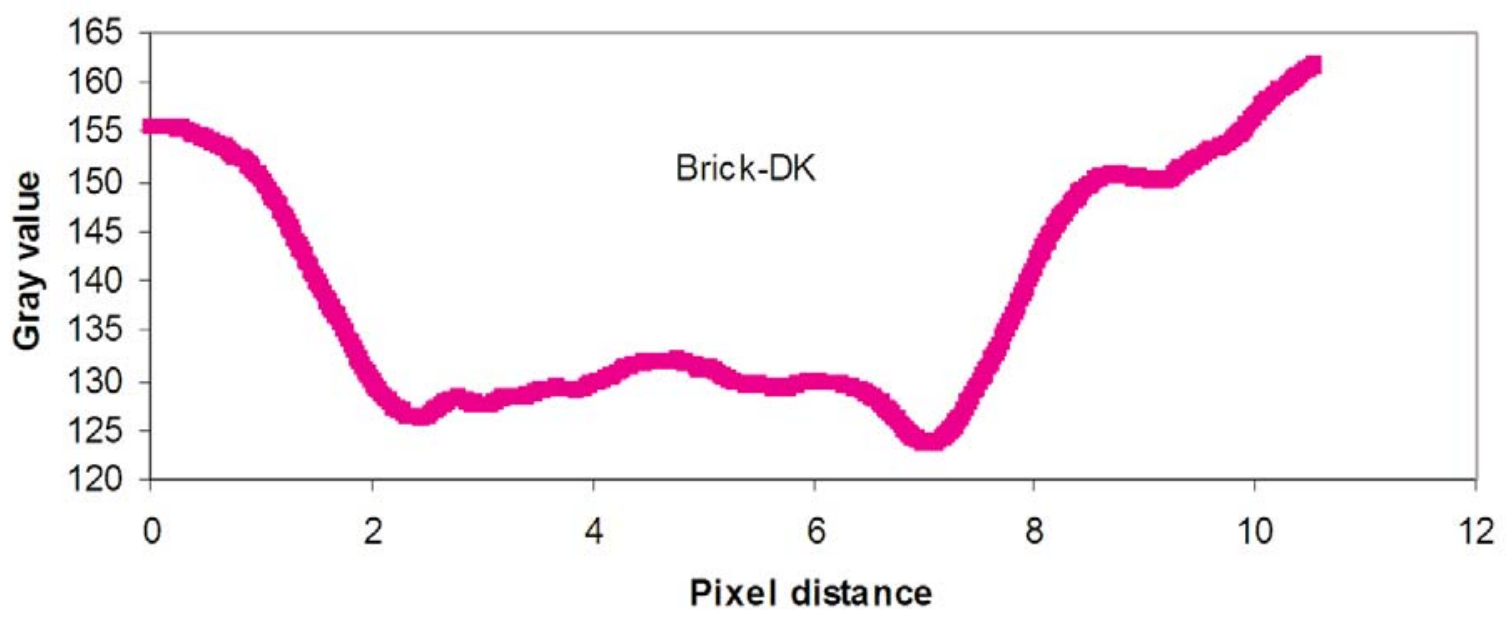

Fig. 5. Gray value vs. pixel distance curve of the radiographic image of the sample

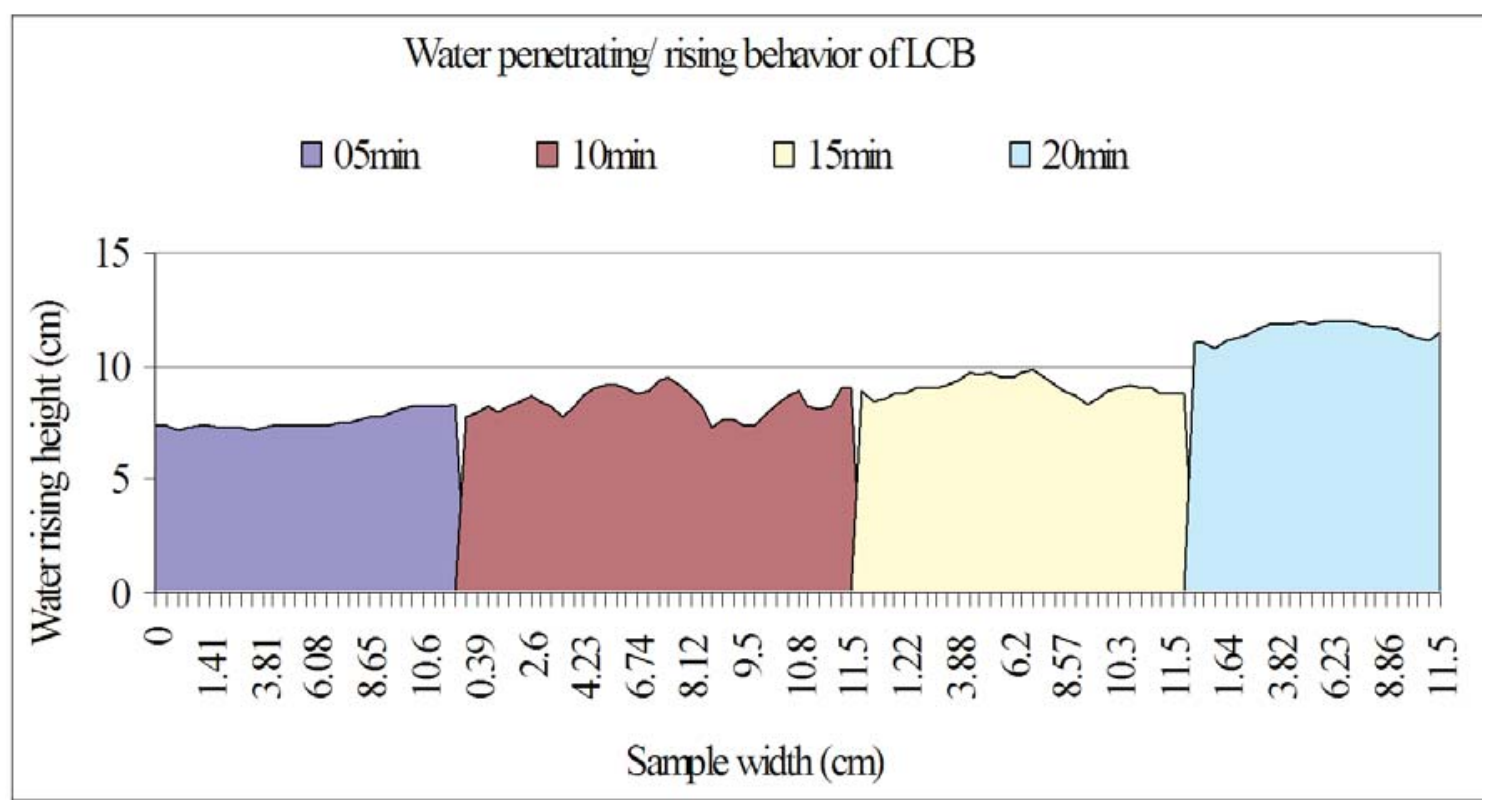

Fig. 6. Water rising behavior of the brick-DK sample at different immersion time 
The gray value has been obtained by drawing line profile of $1056 \times 1600$ pixel area on the radiographic images of an object. Fig. 5 also shows that the middle region is less homogeneous than the two sides. In both sides the porosity of this sample is higher than the middle portion. From this observation it is observed that the contents of the sample is not uniformly distributed all over the sample and indicates the presence of large number of internal porosity. Fig. 6 shows that at 5 minutes water absorption, water is rising $7.4 \mathrm{~cm}$ through the middle part of the sample. It is nearly equal for other two edges. For 10 minutes, water rising through the middle part studied that water absorption depends on submersion time, firing temperature and firing time. High values of water absorption obtained in this study indicate that the clay bricks produced were highly porous. Internal structure of the brick is expected to be intensive enough to avoid intrusion of water (Weng et al., 2003). Few scientists Sanders and Frederic (2011) found a relationship between capillarity and freeze thaw durability. He stated that durability is a function of the pore structure and the nature of the fired bond. On the other hand capillary absorption measures how well water moves through the brick, then it must have some bearing on

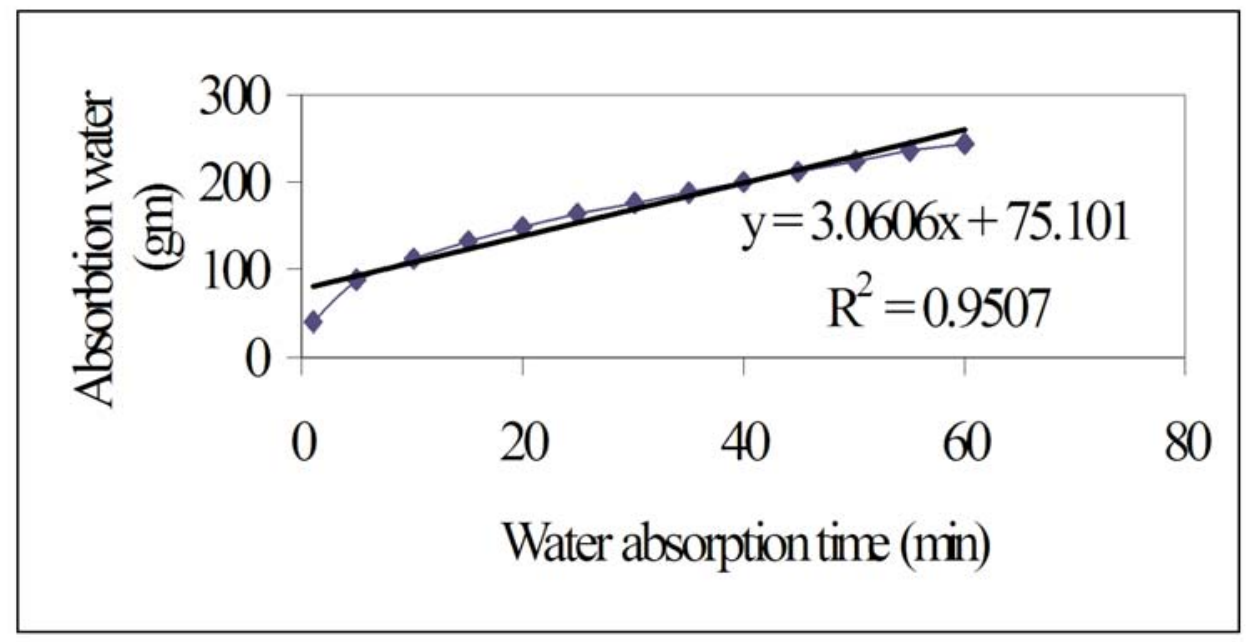

Fig. 7. Water absorption at different immersion time of the sample

is slightly higher than the two edges. In that case water uptake height is $9.4 \mathrm{~cm}$. But in case of 15 and 20 minutes, water penetrating height at middle is $10 \mathrm{~cm}$ and $12 \mathrm{~cm}$, respectively. But at the two edges water uptake is slightly less than the middle part. Few authors studied that water absorption decreased significantly when the temperature increased due to the formation of the amorphous phase at high firing temperature. During the manufacturing time if the clay mixture absorbs more water, brick exhibits a larger pore size, resulting in a lower density (Karaman et al., 2006). Depending on the $\mathrm{H}_{2} \mathrm{O}$ absorption time of brick, observe a difference in capillary absorption was observed (Weng et al., 2003). From the present investigation it also shows that the water rising/penetrating behavior at different immersion time of the brick samples is capillary, almost flat, zigzag shape/behavior. Many authors (TS 704, 1979; TS 705, 1975) the efflorescence potential. Theoretically, the rate of capillary absorption influences the bond between brick and mortar (mixture of lime, water and sand). York dale did not believe that there was a direct relationship between IRA and performance and did not feel that IRA should be included in ASTM specifications. This disagreement is probably related to the lack of information contained in the IRA measurement. Workmanship plays such a large role in the quality of masonry that it is hard to definitively identify the influence of other factors such as rising damp and moisture transfer through masonry.

For a particular type of brick suggests that the connectivity and orientation of pores also play a large part in the movement of water in the pores (Sanders and Frederic, 2011). Fig. 7 indicates that at first 5 minutes water absorption is very 


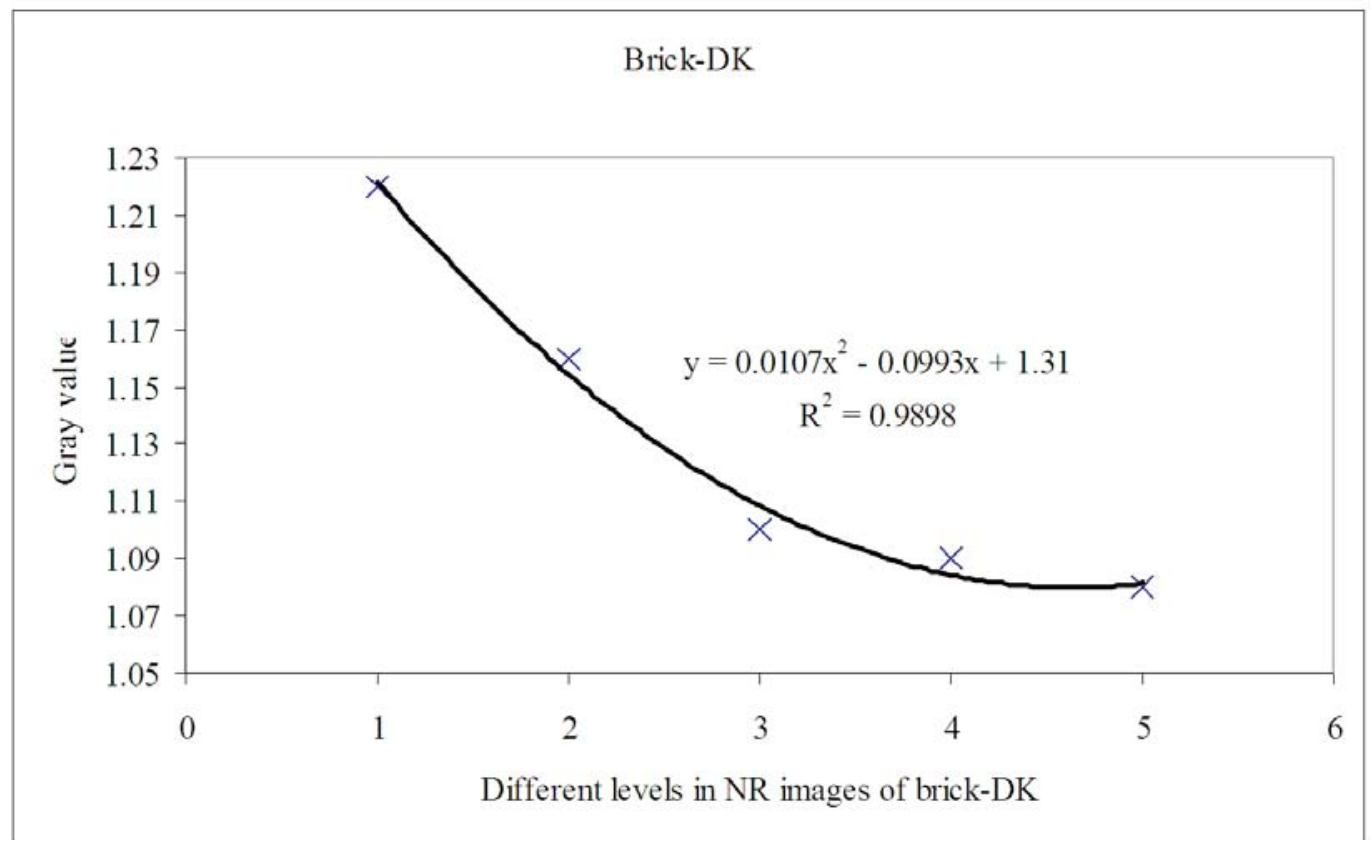

Fig. 8. Densitometric measurement of optical density at different levels of NR image

high. After that the rate of water absorption is very slow. A first-degree polynomial regression equation (Fig. 7) adequately described this relationship $\left(r^{2}=0.95\right)$. Since the effect of immersion time on water absorption is significant. From the optical density measurement (Fig. 8) it is observed that the optical density is different at different levels of the NR images of the sample and it shows far from a straight line.

Optical density is the darkness, or opaqueness, of a transparency film and is produced by film exposure and chemical processing. An image contains areas with different densities that are viewed as various shades of gray. From the optical density measurement (Fig. 8) it is observed that the optical density curve shows far from straight line. From the optical density measurement and porosity, homogeneity, IRA and water penetrating behavior of view it is pointed out that brick-DK is very poor in quality. From the Figs. 4, 6 it can be concluded that the incremental intrusion area for first 5 minutes and the incremental intrusion area correlate to the initial rapid absorption.

\section{Conclusion}

Hand made conventional brick-DK sample is a highly porous brick. Because of irregularity of gray value it is observed that the elemental distribution of the sample is very poor and large number of internal porosity is exist, the incremental intrusion area is very large, initial rapid absorption is very fast. From the analysis it is pointed out that water penetrating/rising through the brick-DK sample is much higher. It is to be noted that the results obtained in this study can only be compared to the properties of bricks produced under similar conditions with similar raw materials.

\section{Acknowledgement}

Authors would like to thanks to the production manager, Lucky conventional bricks (LCB), Dautia, Kalampur, Dhamrai, Dhaka for supplying the sample and to the Ministry of Science \& Technology for their financial aid in order to completion of the research work

\section{References}

Ahsan MH, Islam MN and Alam MK (1995), Proc. of the 2nd International topical Meeting on Neutron radiography system Design and Characterization, Rikkyo University, Japan, Nov. 12-18: 30.

Alam MK (2005), Comparative study of internal defects in ceramic products using CCD-camera based digital neu- 
tron radiography detector. $B J S c i$. Ind. Res., 40(3-4): 169.

Alam MK and Khan MA (2006), Study of water absorption and internal defects in jute reinforced biopol composite using digital neutron radiography technique. $J$. of Bang. Acad. of Sci., 30(1): 29-37.

Alam MK, Khan MA and Lehmann EH (2006), Comparative study of water absorption behavior in Biopol and jute reinforced biopol composite using neutron radiography technique. Journal Rein. Plas. Comp., 25(11): 11791187.

Alam MK, Islam MN and Zaman MA (2007), Study of internal defects and water absorption behavior of single layer Italian tiles using neutron radiography facility of 3 MW TRIGA MARK II research reactor, J. of Bang. Acad. of Sci., 31(2): 213-222.

Berger H (1965), Neutron Radiography, Elsevier, Amsterdam Berger H, ANL-6846 (1964).

Bhatnagar JM and Goel RK (2002), Thermal changes in clay products from alluvial deposits of the Indo-Gangetic plains. Const. Build. Mat., 16(2): 113-122.

Bjelkhagen HI (1993), Silver-halide recording materials. Springer Verlag, pp 128-151.

Bouma AH (1969), Methods for the study of sedimentary structures. A text book, Publisher by John Wiley and Sons and the author Arnold Bouma, New York, (1969) 140 .

Cultrone G, Sebastian E, Elert K, Torre MJ, Cazalla O and Vavarro CR (2004), Influence of mineralogy and firing temperature on the porosity of bricks. J. Euro Ceramic Soc., 13: 621-634.

Hardt PV and Rotterger H (1981) (editor), Neutron radiography hand book, D. Reidel Publishing Company, Dordrecht, Holland.

Harms AA and Wyman DR (1986), Mathematics and physics of neutron radiography, D. Reidel Publishing Company, Holland, 22.
Islam MN, Rahman MM, Ahsan MH, Mollah AS, Ahsan MM and Zaman MA (1995), A Study of Neutron Radiography Parameters at the Tangential Beamport of the 3 MW TRIGA Research Reactor of AERE, Savar. Jahangirnagar University Journal of Sci., 19: 181187.

Islam MN, Alam MK, Zaman MA, Ahsan MH and Molla NI (2000), Application of neutron radiography to building industries. Indian Journal of Pure and Applied Phys., 38: $348-358$.

Islam MN, Rahman MM, Islam SMA and Zaman MA (2000), Neutron radiographic investigation of the quality of some rubber samples. Indian Journal of Pure and Applied Phys. 38: 675-680.

Islam MN, Khan MA, Alam MK, Zaman MA and Matubayashi M (2003), Study of water absorption behavior in wood plastic composite by using neutron radiography technique. Polym. Plast. Technol. and Eng., 42(5): 925-934.

Karaman S, Ersahin S and Gunal H (2006), Firing temperature and firing time influence on mechanical and physical properties of clay bricks. Journal of Scientific \& Industrial research, 65(2): 153-159.

Maithel S, Lalchandani D, Malhotra G, Bhanware P, Uma R, Ragavan S, Athalye V, Bindiya KR, Reddy S, Bond T, Weyant C, Baum E, Thoa VTK, Phuong NT and Thanh TCK (2012), A Roadmap for Cleaner Brick Production on in India, A Shakti Sustainable Energy Foundation and Climate Works Foundation Supported Initiative, April 2012.

Marrusin SL (1985), Interrior fissures and microstructure of shale brick. Am Ceramic Soc. Bull, 64: 674-678. (Turkish Standards Institutions, Ankara) 1979.

Mbumbia L, Wilmars AM and Tirlocq J (2000), performance characteristics of lateritic soil bricks fired at low temperatures: a case study of cameroon: A case study of Cameroon. Const. Build. Mat, 14(3): 121-131.

News report of Bangladesh national paper (2012), The Daily Janakantha 25th June, 2012. 
Norris PM, Brenizer JS, Paine DA and Bostain DA (1996), Measurements of water deposition in aerogel by neutron radiography. Proc. 5th World Conf. on Neutron Radiography, Berlin, Germany, June 17-20, 602.

Part 1 (1986), Manufacturing, Classification, and selection of brick manufacturing. Brick Industry Association, Virginia.

Presertsan S and Theppaya T (1995), A study towards energy saving in brick making. Part 1- Key parameters for energy saving. Int. Energy J., 17(2): 145-156.
Sanders J and Frederic J (2011), The national brick research center, structural clay products division meeting.

TS 704 (1979), Clay bricks (wall tile), Turkish Standards Institution, Ankara.

TS 705 (1975), Solid bricks and vertically perforated bricks, Turkish standards institution, Ankara.

Weng CH, Lin DF and Chiang PC (2003), Utilization of sludge as brick materials, Adv. Environ. Res., 7(3): 679-685.

Received: 02 May 2013; Revised: 18 November 2013; Accepted: 18 December 2013. 